Itinéraires Itinéraires

Littérature, textes, cultures

\title{
Sandman, récit métamorphe : l'espace du chapitre chez Neil Gaiman
}

Sandman as a Shapeshifting Narrative: Neil Gaiman and the Chapter Space

\section{Raphaël Luis}

\section{(2) OpenEdition}

1 Journals

Édition électronique

URL : http://journals.openedition.org/itineraires/7848

DOI : $10.4000 /$ itineraires. 7848

ISSN : 2427-920X

Éditeur

Pléiade

Référence électronique

Raphaël Luis, «Sandman, récit métamorphe : l'espace du chapitre chez Neil Gaiman », Itinéraires [En ligne], 2020-1 | 2020, mis en ligne le 02 octobre 2020, consulté le 14 novembre 2020. URL : http:// journals.openedition.org/itineraires/7848 ; DOI : https://doi.org/10.4000/itineraires.7848

Ce document a été généré automatiquement le 14 novembre 2020.

\section{(c) (i) (9)}

Itinéraires est mis à disposition selon les termes de la licence Creative Commons Attribution - Pas d'Utilisation Commerciale - Pas de Modification 4.0 International. 


\title{
Sandman, récit métamorphe : l'espace du chapitre chez Neil Gaiman
}

\author{
Sandman as a Shapeshifting Narrative: Neil Gaiman and the Chapter Space
}

\author{
Raphaël Luis
}

Face à l'ampleur et l'ambition du roman graphique Sandman, s'étendant sur soixantequinze épisodes et sept ans de publication (1989-1996), l'écrivain et critique Frank McConnell, chargé de rédiger la préface d'un des volumes de la série, se posait la question suivante à propos du créateur de la série, Neil Gaiman :

Gaiman est-il un esprit du genre de Mozart, qui entendait clairement chaque détail d'une composition depuis la première note, ou de celui de Charlie Parker, qui se lançait dans une improvisation et puis tissait ses phrases au hasard en un tout parfait et impeccable? Je n'arrive pas à tirer de lui une réponse nette sur ce point, et peut-être ne le sait-il pas lui-même. (VI, 404¹)

Une telle interrogation provient d'un soupçon : celui que l'écriture en feuilleton, qu'elle soit sous forme de roman ou d'un comic book, ne peut relever que de cette "esthétique divergente » que Régis Messac ([1929] 2011 : 332) lui accordait, l'opposant, du fait de sa recherche de la "multiplicité d'intérêts ", à la fameuse unité de composition et d'effet revendiquée et théorisée par Edgar Allan Poe. Bien que son texte soit extrêmement laudateur à l'égard de Sandman², McConnell ne peut s'empêcher de véhiculer ce soupçon classique à l'encontre de l'écrivain en série, esthétique qui ne commence à être sérieusement étudiée que depuis quelque temps ${ }^{3}$. L'une des limites théoriques de cette méfiance est qu'elle se résume au problème de l'intention de l'auteur et de son plan sur le long terme, sans forcément considérer qu'une analyse esthétique a davantage à se soucier du résultat d'ensemble que de la cohérence a priori de l'œuvre ${ }^{4}$. Le fait d'assister au travers de la publication périodique à l'histoire en train de s'écrire peut entraîner à penser cette segmentation narrative en termes uniquement techniques, ce qui peut expliquer la difficulté de la critique à s'emparer du sujet d'un point de vue esthétique. Raphaël Baroni et Anaïs Goudmand (2017) ont proposé des pistes de réhabilitation poétique de la composition segmentée fort intéressantes lorsqu'il s'agit de se pencher 
sur le rôle esthétique du chapitre dans l'écriture en feuilleton. C'est dans cet esprit que cet article souhaiterait étudier la composition de la production très particulière qu'est Sandman, conçue initialement comme une série périodique avant d'être lue, du fait de son succès critique, comme "la véritable œuvre emblématique de l'émergence du roman graphique ${ }^{5} »$ (Camus 2015, nous traduisons) : la division en chapitres y relève d'une dialectique extrêmement complexe entre le contraint (les exigences éditoriales) et le choisi (la construction d'une architecture seconde, s'émancipant des contraintes et les utilisant à son profit), dialectique qui aboutit à une réelle poétique du comic book comme commentaire et archive de l'histoire des genres narratifs ${ }^{6}$.

\section{Principes d'écritures de Sandman}

2 Sandman n'est pas un récit linéaire ; Gaiman fait avancer sa série à un rythme irrégulier, multipliant les intrigues secondaires, les chemins de traverse et les digressions. Le fil rouge de la série, publiée chez DC Comics à partir de janvier 1989, est l'histoire de Dream, le roi des Rêves, aussi appelé le Sandman. Dream est l'un des Sept Éternels, entités immortelles incarnant certains aspects de la psyché humaine (Dream, Desire, Death, Destruction, Delirium, Despair et Destiny). Emprisonné entre 1916 et 1988 par un occultiste, Dream parvient à s'échapper et revient dans son royaume abandonné, retour qui entraînera toutes sortes de complications.

3 À partir de ce canevas, l'histoire se développe sur soixante-quinze épisodes, qui sont délimités par la taille de vingt-quatre pages imposée par DC Comics. L'organisation de Sandman n'est donc au départ ni un choix esthétique, ni une manière d'affirmer une prétention littéraire, au moins dans un premier temps : elle relève tout simplement des contraintes éditoriales, qui forcent l'auteur à composer avec des unités narratives prédéfinies. Gaiman et les dessinateurs qui ont collaboré à Sandman, sommés par contrat de rendre un nouveau chapitre tous les mois, ont ainsi travaillé comme les feuilletonnistes du XIX ${ }^{e}$ siècle. Gaiman reconnaît bien volontiers la part d'improvisation dans son travail et la nécessité d'écrire Sandman «d'une façon sournoise » (VII, 545) afin d'anticiper les problèmes de cohérence propres à l'écriture en feuilleton. Il explique ainsi avoir fréquemment "cré[é] des personnages jetables» au sein de l'intrigue, dans l'idée de pouvoir les réutiliser si jamais il se retrouvait sans inspiration dans un épisode postérieur :

Je voyais ça comme du jonglage. Je jetais un élément d'histoire en l'air, en sachant que je pourrais le rattraper et le poser dans une scène qui se passerait, disons, douze numéros plus tard. Ou je pouvais ne jamais le rattraper et le laisser simplement suspendu en l'air. (ibid.)

Du point de vue narratif, la dimension feuilletonesque se ressent nettement dans certains enchaînements d'un chapitre à l'autre, Gaiman ayant fréquemment recours au cliffhanger de fin de chapitre qui peut, parfois, être une respiration pour l'auteur avant de trouver ce qu'il va écrire lors du numéro suivant.

Sandman partage ainsi les trois fondements de l'écriture en feuilleton: la contrainte éditoriale, la dimension d'improvisation et l'art du suspense par la mise en attente de la suite de l'histoire. On retrouve là l'idée que le chapitre est une unité narrative provisoire venant de la forme du feuilleton, visant à maintenir la tension de l'intrigue et pouvant donc être lu comme "l'apanage d'une littérature populaire " (Leblond 2012). Or l'originalité de Sandman est de transformer cette structure de feuilleton - qui a tout du récit infini, où les épisodes s'enchaînent dans le but principal de maintenir les 
lecteurs captifs d'une intrigue - en une structure dédoublée: en surplomb de l'organisation en épisodes, qui relève de la consommation immédiate d'un lecteur voulant connaître la suite de l'histoire, Gaiman construit une deuxième structure, bien plus complexe, qui substitue à la régularité rythmique du feuilleton une esthétique fragmentée.

5 Le vocabulaire utilisé par Gaiman pour définir cette double structure peut porter à confusion. Plutôt que de parler d'épisodes, terme employé par la critique pour désigner la livraison périodique d'un récit (ce qu'Ugo Dionne appelle le paradispositif ${ }^{7}$ ), Gaiman parle systématiquement de chapitres lorsqu'il se réfère à ces unités périodiques de vingt-quatre pages. D'où la confusion possible, le terme «chapitre » étant plutôt réservé à «la division du livre en parties, indépendamment de sa publication échelonnée dans le temps» (Colin, Conrad et Leblond 2017:94). Le chapitrage de Sandman - c'est-à-dire l'émancipation par rapport aux contraintes éditoriales de longueur et de rythme des épisodes - s'organise via le recours à ce que Gaiman appelle des arcs narratifs, qui englobent plusieurs épisodes et proposent une réelle unité de sens.

6 Ce redoublement du chapitrage est en un sens une conséquence directe de la longueur (près de deux mille planches) de Sandman, qui pousse Gaiman à fournir une aide au lecteur pour se repérer dans une œuvre d'une grande complexité narrative ; mais elle est aussi un signe de la dimension esthétique que l'auteur a voulu donner à un genre qui, à la fin des années 1980, n'est encore que peu reconnu par les instances de légitimation critiques ${ }^{8}$. Sandman est un des rares exemples d'œuvre qui, du fait de la reconnaissance critique qu'elle a reçue, semble avoir changé de genre en cours de publication : comme le dit Bissette (2008: 151, nous traduisons), «Sandman est passé au fur et à mesure d'une publication périodique à un roman graphique à plusieurs chapitres ${ }^{9}$ ». À l'image de certains romanciers du xix siècle effaçant les traces du découpage en épisodes lors de la parution chapitrée de leur ouvrage (Colin, Conrad et Leblond 2017: 151-152), Gaiman met en concurrence deux structures, celle de la division éditoriale en épisodes et celle du choix esthétique du chapitrage, à ceci près que l'opposition épisode/chapitre devient chez lui un couple chapitre/arc narratif. Cela étant, Sandman ne fait pas de cette concurrence un processus d'effacement du feuilleton: les épisodes, au contraire, sont une unité essentielle de l'organisation que cherche à bâtir l'auteur. C'est pourquoi nous conserverons, dans les lignes qui suivent, la terminologie de Gaiman, pour ce qu'elle révèle de la volonté de ce dernier d'unir parution périodique et chapitrage dans une même temporalité.

\section{Arcs narratifs, chapitres, récits indépendants : une architecture complexe}

7 Vue avec le recul d'un lecteur ayant lu les soixante-quinze chapitres, l'architecture de Sandman évoque une version déformée des séries télévisées telles qu'on les produit aujourd'hui. Dix grands arcs narratifs remplissent peu ou prou le rôle des saisons, tandis que les chapitres jouent celui des épisodes. Cependant, là où la série télévisée est le plus souvent contrainte à la fois du point de vue de la longueur des saisons et de celle des épisodes, Gaiman prend une totale liberté quant à la première injonction. La fonction esthétique première de son chapitrage est en effet de briser la linéarité 
répétitive de l'enchainement des épisodes et de travailler le rythme de son récit. La structuration en arcs narratifs y participe de plusieurs façons :

8 1. Chacun des arcs narratifs de Sandman a une atmosphère différente, due à la volonté de Gaiman de modifier l'ambiance et les enjeux de chaque unité narrative qu'il propose. D'où un désir très net de mettre en contraste la régularité immuable des chapitres avec la taille systématiquement dissemblable des arcs narratifs; si ces derniers ont une durée moyenne de sept ou huit chapitres, ils peuvent aussi en comporter quatre (Dream Country) ou treize (The Kindly Ones), selon les besoins du récit. Gaiman modifie également régulièrement l'ambiance visuelle de Sandman. Commencée avec Mike Dringerberg et Todd Klein au dessin, la série voit peu à peu apparaitre de nouveaux artistes, Gaiman finissant par choisir un dessinateur par arc narratif selon l'atmosphère qu'il souhaite créer ; pour ne prendre qu'un exemple, Shawn McManus a été choisi pour illustrer A Game of You parce que Gaiman avait besoin d'un dessinateur «qui sache œuvrer à la fois dans le dessin caricatural mignon et dans l'horreur réaliste, et les mêler sans solution de continuité » (III, 394) ${ }^{10}$.

9 2. Ces arcs ne sont pas nécessairement des unités de récit: si le récit linéaire de l'histoire de Dream compose l'essentiel d'entre eux, d'autres sont des compilations de contes fonctionnant comme des parenthèses dans la trame générale. Les arcs narratifs 3 (Dream Country), 6 (Fables and Reflections) et 8 (World's End) n'ont ainsi pas de rapport direct avec le récit principal et servent avant tout à enrichir l'univers fictionnel de la série ; leur cohérence interne est dès lors une question thématique plus que narrative, chacune des histoires formant l'arc étant une déclinaison d'un sujet plus général. Dream Country ne comporte ainsi que des histoires se déroulant dans le monde du rêve, mais où le Sandman n'est qu'un personnage secondaire apparaissant dans les rêves et la vie de chats, d'un écrivain raté ou de Shakespeare, ce qui permet de briser la linéarité chronologique du récit.

10 3. L'alternance est le maître mot de Sandman : alternance entre arcs longs et arcs courts, entre intrigue linéaire et récits secondaires mais aussi, de façon moins intuitive, entre histoires masculines et histoires féminines. Sandman, affirme Gaiman dans un entretien, «a été conçu pour passer d'histoires masculines à des histoires féminines " (III, 393), la division en arcs narratifs permettant cette alternance. Ce mouvement de balancier concerne principalement les arcs dans lesquels domine l'histoire linéaire : les arcs 1,4 et 7 sont à dominante "masculine », au sens où, comme le dit Gaiman, « le recueil a un héros masculin, le Sandman, qui triomphe de divers défis difficiles » (ibid.); les arcs 2, 6 et 9 sont à dominante « féminine » car Dream y est plus en retrait, laissant la place à des protagonistes féminins. Cette répartition genrée a une double ambition : d'une part, elle contribue à la variété du rythme du récit, en éloignant régulièrement le lecteur de l'histoire de Dream ; d'autre part, elle est un des fondements de l'ambition de Gaiman d'ouvrir les comic books à un public plus large que son lectorat habituel, notamment en attirant des lectrices bien moins consommatrices d'histoires de superhéros que leurs homologues masculins.

11 4. L'organisation en arcs narratifs permet enfin à Neil Gaiman de détourner l'usage du chapitre, en extrayant ce dernier du flux linéaire du récit. Lorsqu'un arc narratif est composé de chapitres s'enchaînant selon le rythme chronologique du récit, Gaiman interrompt fréquemment ce dernier en introduisant un chapitre indépendant qui crée une parenthèse dans le récit. C'est ainsi le cas avec le chapitre "Men of Good Fortune " de The Doll's House (épisode 13 de la série) ou le «Chapter 4 » de Season of Mists 
(épisode 24). Dans un entretien, Gaiman explique que ce processus, qu'il a essayé de ne pas systématiser pour ne pas " devenir prévisible ", sert de commentaire métatextuel plus ou moins dissimulé sur l'histoire en train d'être racontée, le chapitre indépendant fonctionnant à la fois comme résumé en miniature et problématisation de l'ensemble de l'arc narratif (VII, 546).

12 À partir de ces différents éléments qui illustrent le processus de dédoublement du chapitrage effectué par Neil Gaiman, il est possible de représenter l'architecture de Sandman, sous forme simplifiée, par le schéma suivant ${ }^{11}$ :

Tableau 1 : Composition narrative de Sandman

\begin{tabular}{|c|c|}
\hline Histoire linéaire (Dream) & Chapitres indépendants \\
\hline \multicolumn{2}{|c|}{ Arc 1 Preludes and Nocturnes } \\
\hline \multicolumn{2}{|l|}{ \# 1-8 } \\
\hline \multicolumn{2}{|l|}{ Arc 2 The Doll's House } \\
\hline & \# 9 « Tales in the Sand» \\
\hline \multicolumn{2}{|l|}{ \# 10-12 } \\
\hline & \# 13 « Men of Good Fortune » \\
\hline \multicolumn{2}{|l|}{ \# 14-16 } \\
\hline \multicolumn{2}{|l|}{ Arc 3 Dream Country } \\
\hline & \# 17-20 \\
\hline \multicolumn{2}{|l|}{ Arc 4 Season of Mists } \\
\hline \multicolumn{2}{|l|}{ \# 21-23 } \\
\hline & \#24 « Chapter 4 » \\
\hline \multicolumn{2}{|l|}{ \# 25-28 } \\
\hline \multicolumn{2}{|c|}{ Arc 5a Fables and Reflections : Distant Mirrors } \\
\hline & \# 29-31 \# 50 « Ramadan » \\
\hline \multicolumn{2}{|l|}{ Arc 6 A Game of You } \\
\hline \multicolumn{2}{|l|}{ \# 32-37 } \\
\hline \multicolumn{2}{|c|}{ Arc 5 b Fables and Reflections : Convergence } \\
\hline & \# 38-40 \\
\hline \multicolumn{2}{|l|}{ Arc 7 Brief Lives } \\
\hline \multicolumn{2}{|l|}{ \# 41-49 } \\
\hline \multicolumn{2}{|l|}{ Arc 8 World's End } \\
\hline & \# 51-56 \\
\hline \multicolumn{2}{|l|}{ Arc 9 The Kindly Ones } \\
\hline \# 57-69 & \\
\hline
\end{tabular}




\begin{tabular}{|l|l|}
\hline$\# 70-72$ & \\
\hline & $\# 73-75$ \\
\hline
\end{tabular}

13 En construisant une architecture d'une telle complexité, Gaiman crée la possibilité de plusieurs types de lectures : la lecture linéaire suit le rythme éditorial des chapitres, mais sans être soumise à l'unique enchaînement répétitif construit par les effets de suspense ; un lecteur ne voulant pas lire l'ensemble de la série peut se contenter d'un seul arc narratif, puisque chacun d'eux a une unité narrative, esthétique ou thématique; enfin, un lecteur ayant déjà lu l'ensemble de la série peut la relire de manière moins linéaire en essayant de comprendre les continuités, les échos et les variations apportées par le second chapitrage. La complexité architecturale de Sandman est donc, en définitive, une manière d'élargir les manières de lire un comic book et, par conséquent, d'élargir le lectorat possible du genre.

\section{Les chapitres, archives des genres}

Ce système de double chapitrage permet à Gaiman de donner une utilité esthétique à des chapitres qui, sans le rattachement à un arc narratif, en seraient réduits à leur existence d'épisodes périodiques. L'utilisation des titres en est un premier exemple : la plupart des arcs narratifs proposent une unité dans la mise en titre, de façon à ce que l'indépendance de chacun d'entre eux se répercute dans la manière même dont les chapitres sont présentés.

Dans Season of Mists, Gaiman souhaite ainsi donner à l'arc narratif l'atmosphère d'une histoire " grande, victorienne et baroque ; très apprêtée et ornementée » (II, 343). Pour ce faire, il recourt à ce que Genette (1987: 68-69) appelle des "titres-arguments", classiques dans les romans $d u x I_{I I}{ }^{e}$ et du xIx siècle, qui annoncent par de longues phrases ce que le chapitre va contenir. Le chapitre 1 est introduit par le titre suivant: "Où le Maître des Rêves se prépare à visiter les royaumes infernaux; des adieux sont prononcés; un toast est porté ; et en Enfer, l'Adversaire règle de son côté certains préparatifs » (II, 144). La police employée pour écrire ces titres ressemble à celle employée dans des ouvrages de l'ère victorienne, avec des ornements et des ligatures qui cherchent à donner cette impression d'ancienneté. Dans A Game of You, dont l'intrigue est beaucoup plus contemporaine que Season of Mists, qui parlait de la relation de Dream et de Lucifer, Gaiman change complètement d'inspiration et donne cette foisci aux chapitres des titres de chansons, plus ou moins modifiées selon ses besoins. De la comédie musicale ("Slaughter on Fifth Avenue», "Lullabies of Broadway») à l'imitation de la chanson traditionnelle écossaise («Over the Sea to Sky»), en passant par des titres de Creedence Clearwater Revival («Bad Moon Rising»), Lou Reed ("Beginning to See the Light») ou Elvis Costello («I Woke Up and One of Us Was Crying »), les titres des chapitres fournissent une unité paratextuelle à l'arc narratif, confirmant au lecteur qu'il a bien changé de genre d'histoire par rapport à l'arc précédent. Dans l'arc Brief Lives, dont le personnage central est Delirium, la petite sœur de Dream, les titres sont entièrement déconstruits, comme si l'esprit du délire envahissait le récit. La mention « chapitre » disparaît, au profit d'un simple numéro suivi d'une série de titres obscurs, évoquant davantage la poésie contemporaine la plus expérimentale qu'un paratexte classique. Ainsi du premier chapitre, titré de la façon 
suivante : «Un : Une fleur pour une dame - La pluie sur le seuil - Pas sa sœur - Vouloir ou pas - Vu du revers des miroirs - Journal de l'année de peste - "Le numéro que vous avez composé..." » (IV, 104). Dans Distant Mirrors, les quatre chapitres, qui traitent tous "de la responsabilité des rois » (III, 409), sont reliés entre eux par des titres sous forme de dates ("Three Septembers and a January », « August », « Thermidor ", « Ramadan »).

Ces variations sur les titres des chapitres laissent entrevoir un processus plus global, qui est un point nodal de l'ambition de Sandman : à partir de l'arc 4 (Season of Mists), chaque arc narratif de la série est rattaché à une tradition générique différente, que l'organisation en chapitres illustre et met en exergue. S'il est difficile d'associer Sandman à un genre définitif, c'est parce que la série fonctionne comme un commentaire sur les différents types de récits, commentaire mis en évidence par l'architecture interne de l'ensemble du récit. Si Season of Mists est, comme souligné cidessus, un arc baroque et victorien, A Game of You est quant à lui une imitation des romans de fantasy centrés sur un personnage de jeune fille : son personnage central, la princesse Barbie, y est un mélange entre la Alice de Lewis Carroll et la Dorothy de The Wizard of $\mathrm{O} z$, accompagnée comme ces dernières d'un étrange bestiaire de créatures dotées de parole. Gaiman dit au demeurant avoir écrit cet arc comme "une sorte d'histoire anti-fan sur la nature des milieux faniques et les raisons pour lesquelles les gens aiment la fantasy » (III, 394). Distant Mirrors emprunte de son côté à une tout autre tradition, celle des récits de vies célèbres, Gaiman s'inspirant notamment de Suétone pour l'épisode "August» et ayant eu effectué un vrai travail de documentation historique pour les épisodes sur Robespierre et sur l'empereur Norton (IV, 409). À l'intérieur même des arcs narratifs, Gaiman se permet des détours pour consacrer un chapitre à un genre particulier : ainsi de l'épisode 6 de "Preludes and Nocturnes », qui est un huis-clos d'horreur, et du «Chapter 4 » de Season of Mists, qui relève à la fois de l'histoire de revenants et de la satire du système scolaire anglais.

Ce souci de parcourir dans Sandman la diversité des genres narratifs pousse même Gaiman à consacrer un arc narratif entier à la succession de divers récits de genre. World's End, qui précède le dénouement raconté dans The Kindly Ones est ainsi un recueil d'histoires que Gaiman voyait comme sa "dernière chance d'explorer des genres divers » (IV, 366). Pour ce faire, il fait appel à une première tradition narrative, les contes intégrés dans un récit-cadre. Plus que les Mille et une nuits, ce sont ici les Canterbury Tales qui servent de référence assumée : comme chez Chaucer, le point de départ est la rencontre de voyageurs dans une auberge, chacun de ces derniers en venant à raconter une histoire pour divertir les autres ${ }^{12}$. Chaque chapitre de World's End est constitué d'une de ces histoires, toutes empruntées à un genre différent: on y trouve successivement un conte à la Lovecraft, une histoire de cape et d'épée, un récit d'aventures initiatiques inspiré de Kipling et un pastiche des fables morales sur la réussite personnelle écrites par Horatio Alger au XIX ${ }^{e}$ siècle. Après avoir entendu toutes ces histoires, une des femmes de l'assistante, Charlene, se plaint du manque de variété de ce qui est raconté :

Pourquoi ces histoires-là ? Toutes celles que nous avons entendues, ce sont toutes des récits de garçons, non? Nous avons eu une aventure de cape et d'épée, un récit de mer, une histoire de gangster, un horrible conte d'enterrement et même une petite histoire de fantôme. Ce sont des récits de garçons. Voilà ce que c'est. $(\mathrm{V}, 178)$

La remarque est à l'évidence un commentaire de Neil Gaiman lui-même sur l'impossible tâche consistant à répertorier tous les genres narratifs connus à l'intérieur d'une histoire dont le nombre de chapitres reste limité ; c'est aussi, dans la continuité de sa 
volonté d'alterner entre histoires masculines et histoires féminines, une réflexion sur la domination du masculin dans la tradition narrative occidentale. Comme le dit Charlene dans la suite de sa tirade, «il n'y avait aucune vraie femme dans [ces] histoires. Nous sommes juste de jolies silhouettes, du décor, à aimer, perdre, éviter, à qui obéir... » (ibid.). Malgré ce commentaire sur la limite de son entreprise, il apparaît clairement que Sandman est dans l'esprit de Gaiman une archive de l'ensemble des genres narratifs, archive recueillie dans une forme qui, parce qu'elle n'appartient pas directement à la littérature telle qu'elle est définie habituellement, possède la souplesse structurelle nécessaire pour soutenir cette ambition. Le mélange de malléabilité esthétique et de rigidité éditoriale du comic books est la condition de la réussite du projet de Sandman.

\section{Sandman comme pièce tragique}

Bien comprendre cette adéquation entre le projet esthétique de Sandman et la forme du comic book suppose de s'attarder sur ce qui, dans la série de Gaiman, est propre au média de la bande dessinée. Nous avons jusqu'à maintenant principalement étudié l'utilisation du chapitre dans Sandman du point de vue de l'organisation structurelle ou de l'identité générique. Or la particularité esthétique de la bande dessinée qualifiée de littéraire suppose, comme l'a montré Baetens (2009), de ne soustraire de l'analyse ni le rapport au texte, ni celui à l'image - pour le dire autrement, de ne l'étudier ni comme du cinéma, ni comme du dessin, ni comme un simple récit.

Cette polyvalence de la bande dessinée apparaît très nettement dans l'arc narratif le plus développé de Sandman, The Kindly Ones. Composé de treize chapitres, cet arc est le dénouement de l'histoire de Dream, poursuivi par les Parques (les «bienveillantes » du titre) pour avoir tué son fils Orphée. Les titres de chapitres y sont d'une grande sobriété : écrits en petits caractères, ils se composent uniquement du titre de l'arc narratif suivi du numéro du chapitre. C'est que leur appartenance à un même arc y est, cette fois-ci, suggérée visuellement par un motif qui est à la fois structurel et narratif. Les chapitres sont en effet, dans The Kindly Ones, liés entre eux par le dessin : chaque case d'ouverture d'un chapitre est traversée par un fil (pelote de laine, fil téléphonique, etc.) qui rend concrète l'appartenance de chacun des épisodes à un même arc narratif. Comme le dit Gaiman lui-même, ces fils sont « accompagnés d'un texte commentant le point où nous en étions arrivés dans l'histoire » (VI, 395), comme pour répondre à l'inquiétude d'un lecteur déboussolé par la longueur de cet arc narratif conclusif, composé de dizaines d'échos des chapitres précédents. Le premier chapitre s'ouvre par les questions : "Est-ce prêt ? Tu as fini ? ", qui entraînent une discussion entre les trois Parques sur ce que signifie finir une histoire. Les trois pages de la discussion (VI, 21-23) sont composées de cases traversées par le fil que les déesses tissent, avant qu'un gros plan ne montre l'une d'entre elles coupant le fil avec ces mots: «Faut bien finir un jour.» Tout lecteur ayant lu les cinquante-six chapitres précédents a compris que l'action de couper le fil annonce la fragilité du fil de la vie de Dream, et que le motif visuel va nous informer à chaque début de chapitre sur la distance qui nous sépare de la mort du personnage principal - et, donc, de la fin de Sandman. Ce faisant, Gaiman renonce au principe du cliffhanger, puisqu'il annonce discrètement le dénouement. Il se permet par conséquent de jouer avec les attentes du lecteur, allant totalement à 
rebours du principe de suspense de l'écriture sérielle : ce n'est plus l'événement final qui est attendu comme un mystère, mais la façon dont l'histoire va y arriver.

Chaque chapitre de The Kindly Ones devient une manière de repousser la fin inévitable de la série, ironiquement commentée par l'auteur lui-même dans l'association texte/ image de chaque première case. Le chapitre 2 commence ainsi par une case montrant un fil de téléphone accompagné de la réplique : «Eh bien? Combien de temps ça va prendre?» (VI, 47); dans le chapitre suivant, un cordon d'argent est surplombé par le dialogue suivant: «Je crois que ça va être plus grand que j'avais prévu. - Peu m'importe. Aussi grand qu'il le faudra " (VI, 73). Gaiman commente ici ses propres doutes sur la durée de l'arc narratif, censé au départ être composé de six chapitres, mais dont la taille augmente au fur et à mesure de la rédaction: «j'aimerais être certain que je fais ce qui convient» (VI, 99), dit ainsi un des personnages dans la première case du chapitre 4 , devant un fil électrique ; « ça avance très lentement, mais ça avance » (VI, 125), dit un autre dans la case d'ouverture du chapitre 5 , où le fil est une toile d'araignée; «j'aurais jamais pensé arriver là un jour » (VI, 177), avoue un troisième au début du chapitre 7 , qui reprend le motif du fil téléphonique. Dans le chapitre 9, on retrouve un gros plan sur la main d'une des Parques, tenant un ciseau s'apprêtant à couper un nouveau fil et annonçant : « Presque l'heure » (VI, 229). Gaiman utilise ici au maximum les possibilités techniques de la bande dessinée : le découpage en chapitres est le vecteur d'un commentaire métatextuel exprimé par l'association du texte et de l'image, sans même avoir recours à la présence d'un récitant ${ }^{13}$.

Ce procédé est aussi, comme toujours dans Sandman, un commentaire générique sur l'histoire se déroulant sous les yeux du lecteur. Si l'architecture des épisodes précédents a permis de mettre en valeur la dimension d'archive de la série, la composition de The Kindly Ones ramène le lecteur à la signification de cette archive. Donner à l'arc narratif le nom des Parques et l'organiser autour de l'image du fil est à l'évidence une manière de rattacher la fin de Sandman à la tragédie. Non seulement la représentation des Bienveillantes comme trois sorcières rassemblées autour d'un chaudron renvoie au début de Macbeth, mais Gaiman parsème aussi l'histoire de références aux tragédies jacobéennes: The Kindly Ones s'ouvre sur deux citations tirées de Cupid's Revenge de Beaumont et Fletcher et de The White Devil de Webster, dramaturge dont Gaiman dissimule certaines phrases dans les dialogues (VI, 394). La mise en attente de la mort de Dream par la longueur de l'arc narratif n'est qu'une façon de recréer chez le lecteur le sentiment d'inéluctabilité suscité par le genre tragique. C'est aussi, à l'évidence, un autre exemple de la volonté de Gaiman de briser les habitudes du lectorat des comic books, en lui retirant le confort de l'invincibilité du héros destiné à pouvoir être éternellement réutilisé dans de nouvelles histoires.

En choisissant un dénouement tragique, Gaiman offre au lecteur la possibilité de relire l'intégralité de la série à l'aune du destin de Dream. Apparaît alors, dans cette architecture si complexe, l'impression que les soixante-quinze chapitres de la série peuvent être vus comme autant de scènes d'une grande pièce de théâtre où les arcs narratifs jouaient le rôle des actes. Après l'acte d'exposition (Preludes and Nocturnes), construit autour du thème classique du héros revenant en ce royaume, les arcs suivants peuvent être vus comme les solutions cherchées par Dream pour revenir à l'équilibre. Le nœud tragique se situe dans Brief Lives, lorsque Dream décide d'aller voir son fils Orphée décédé à cause de lui. Gaiman témoigne du changement qui s'est fait en lui à partir de ce moment : 
C'est un signal : il n'y a plus de retour en arrière possible, désormais. Nous sommes entrés dans le troisième acte ; l'histoire va vers sa fin. À partir de ce moment, écrire Sandman est devenu très bizarre, parce qu'une partie de moi sentait que c'était déjà terminé. Jusque-là, j'aurais pu éviter la fin que j'avais prévue ; en fait, j'avais établi plusieurs issues de secours, au cas où j'aurais changé d'avis sur la direction que la série allait emprunter. Mais le chapitre 7 de Brief Lives représente ce moment où le roi Lear se retourne et dit: «Bon, alors, laquelle de vous trois m'aime le plus, mes filles? » $(\mathrm{IV}, 421)$

Cette lecture théâtrale de la composition de Sandman paraît d'autant plus convaincante que le tout dernier chapitre de la série est consacré à l'aide que Dream aurait fourni à Shakespeare pour écrire The Tempest, où le personnage de Prospero est souvent commenté comme un alter ego du dramaturge faisant ses adieux au théâtre : le rideau tombe sur la scène comme il tombe sur Sandman, qui se conclut après dix actes, soixante-quinze scènes et de nombreux changements de décors. En créant cette structure souple en arcs narratifs sans jamais éliminer la contrainte éditoriale du feuilleton, Gaiman permet cette souplesse de l'interprétation : puisque le lecteur assiste à une structuration qui n'est pas a posteriori mais dans le flot même de la parution périodique, il accompagne l'œuvre dans ses changements de forme, ses passages d'un genre ou d'un type de représentation à un autre.

\section{Conclusion}

Parce qu'il a été créé comme une série périodique soumise aux lois de la publication en feuilleton, et que l'ambition de son auteur et son succès critique l'ont déporté vers un genre éditorial, le graphic novel, dont l'esprit est « une tentative de dépassement des formes populaires » (Baroni 2018:6), Sandman est un exemple fascinant de la légitimité qui est accordée ou non au chapitre : l'organisation de la série de Neil Gaiman semble en effet devenir littéraire à partir du moment où elle s'émancipe des carcans éditoriaux de DC Comics pour créer sa propre architecture, transformant la succession d'épisodes à la longueur imposée en arcs narratifs composés de chapitres, rapprochant ainsi la forme du comic book de celle du roman. Cela étant, analyser l'architecture de Sandman uniquement en termes de recherche de légitimité culturelle ou de prétention littéraire formelle serait sous-estimer la fonction esthétique de la contrainte dans le travail de Gaiman. Ce dernier ne court-circuite pas les épisodes ; il les transforme en chapitres, les utilisant comme un terrain d'expérimentation pour construire avec Sandman une archive des genres littéraires, le jeu sur les rythmes, les durées, les échelles de construction et de narration ayant comme but de faire advenir un récit métamorphe, dont le genre est décidé autant par l'auteur que par le lecteur. En comprenant que le chapitre n'a pas seulement une fonction rythmique (Dionne 2008) mais est aussi un vecteur d'identification générique, Gaiman parie sur l'autonomie du lecteur et lui offre la possibilité de reconnaître dans Sandman le ou les genres qu'il affectionne, sans pour autant sacrifier la présence d'une intrigue linéaire donnant une unité à l'ensemble. Le chapitre, dans Sandman, devient alors un facteur de liberté : liberté de lire en série, de lire dans un genre ou de lire dans tous les genres possibles. Il est cet espace que le lecteur peut plier et déplier à son aise, pour construire cette œuvre par superposition dont semble rêver Gaiman, où toute référence sérielle peut être lue de manière savante, où toute histoire masculine a son versant féminin ; il est donc, en définitive, un espace de réconciliation narrative. 


\section{BIBLIOGRAPHIE}

Baetens, Jan, 2009, « Littérature et bande dessinée. Enjeux et limites », Cahiers de narratologie, $\mathrm{n}^{\circ}$ 16, [En ligne], DOI : 10.4000/narratologie.974.

Baetens, Jan, Frey, Hugo et Tabachnik, Stephen E., 2018, The Cambridge History of the Graphic Novel, Cambridge, Cambridge University Press.

Baroni, Raphaël et Goudmand, Anaïs, 2017, « De l'épisode au chapitre : la fonction esthétique de la segmentation narrative ", dans C. Colin, T. Conrad et A. Leblond (dir.), Pratiques et poétiques du chapitre du XIX ${ }^{e}$ siècle au XXI ${ }^{e}$ siècle, Rennes, Presses Universitaires de Rennes, p. 119-133.

Baroni, Raphaël, 2018, « Le chapitrage dans le roman graphique américain et la bande dessinée européenne : une segmentation précaire ", Cahiers de narratologie, $\mathrm{n}^{\circ} 34$, [En ligne], DOI : 10.4000/ narratologie.8594

Bernard, Rémi, 2016, « Conteurs de bulles et de cases dans Sandman », Quaderno 08, supplément à « AGON », Rivista Internazionale di Studi Culturali, Linguistici e Letterari, nº 11, p. 482-509.

Bissette, Stephen R., Golden, Christopher et Wagner, Hank, 2008, Prince of Stories: The Many Worlds of Neil Gaiman, New York, St Martin's Press.

Camus, Cyril, 2015, « Neil Gaiman's Sandman as a Gateway from Comic Books to Graphic Novels », Studies in the Novel, vol. $47, \mathrm{n}^{\circ} 3$, p. 308-318.

Colin, Claire, Conrad, Thomas et Leblond, Aude (dir.), 2017, Pratiques et poétiques du chapitre du XIXe siècle au XXI ${ }^{e}$ siècle, Rennes, PUR.

Dionne, Ugo, 2008, La voie aux chapitres, Paris, Seuil.

Genette, Gérard, 1987, Seuils, Paris, Seuil.

González Echevarría, Roberto, 2000, Mito y archivo: una teoría de la narrativa latinoamericana, Mexico, Fondo de cultura económica.

Groensteen, Thierry, 2001, Bande dessinée et narration. Système de la bande dessinée 2, Paris, PUF.

Kelleter, Frank, 2017, Media of Serial Narrative, Columbus, The Ohio State University Press.

Leblond, Aude, 2012, « Le chapitre invisible? », posté le 19 juin 2012, consulté le $1^{\text {er }}$ décembre 2018, [En ligne], https://chapitres.hypotheses.org/62.

Letourneux, Matthieu, 2017, Fictions à la chaîne. Littératures sérielles et culture médiatique, Paris, Seuil.

Messac, Régis, [1929] 2011, Le « Detective Novel » et l'influence de la pensée scientifique, Paris, Les Belles lettres.

Revaz, Françoise, 2013, « Le récit suspendu : un genre narratif transmédial », dans M. Monte et G. Philippe, Genres et textes. Déterminations, évolutions, confrontations, Lyon, Presses Universitaires de Lyon, p. 117-134.

Revaz, Françoise, 2017, «L'épisode hebdomadaire des histoires “à suivre" : une forme de "chapitre minimal” ? ", dans C. Colin, T. Conrad et A. Leblond (dir.), Pratiques et poétiques du chapitre du XIX ${ }^{e}$ siècle au XXI ${ }^{e}$ siècle, Rennes, PUR, p. 135-149. 


\section{Corpus}

Gaiman, Neil, 2012-2016, Sandman, intégrale en sept volumes, Paris, Urban Comics.

\section{NOTES}

1. Pour ne pas surcharger le texte, nous ferons ainsi référence aux sept tomes de l'édition française de Sandman (Gaiman 2012-2016), avec le numéro du tome suivi du numéro de la page.

2. Il écrit ainsi que Gaiman est « un conteur virtuose et, sans doute, un génie » et que sa série est « une des histoires les plus stupéfiantes du dernier demi-siècle - dans n'importe quel média » (VI, 402).

3. Voir notamment Letourneux (2017) et Kelleter (2017), ainsi que Revaz (2013) sur le genre du « récit suspendu ».

4. On retrouve là la concurrence entre deux analyses possibles des récits périodiques, présentées dans Revaz (2017: 137) : « une analyse après-coup, une fois que le récit est enfin arrivé à son terme » et "une analyse plus dynamique qui tienne compte de la dimension sérielle (temporelle et séquentielle) tant de la production que de la réception de ces récits".

5. Le terme de graphic novel commence à s'imposer à partir de la parution de A Contract with God: A Graphic Novel de Will Eisner en 1978. Sandman fait partie de la première vague de romans graphiques rencontrant un succès commercial et critique, à la suite d'une année 1986 marquée par la parution de Maus de Spiegelman et des productions de DC Comics Watchmen (Alan Moore et Dave Gibbons) et The Dark Knight Returns (Frank Miller). Voir Baetens, Frey et Tabachnik (2018).

6. Nous employons ici «archive » au sens défini par Roberto González Echevarría à la suite de Michel Foucault: la capacité d'un texte "à inclure toutes les modalités narratives du passé, comme une sorte de mémoire active, de réservoir de possibilités narratives » (González Echevarría 2000 : 26. Nous traduisons).

7. Dionne (2008).

8. Le label Vertigo, créé en 1993 par DC Comics et dans lequel sera publié Sandman à partir du numéro 47 , est justement une réaction au succès public et critique rencontré par les comic books littérairement ambitieux écrits par les auteurs britanniques Alan Moore, Grant Morrison ou Neil Gaiman, entre autres. Nous renvoyons ici au colloque "1993-2018 : Vingt-cinq ans de Vertigo ", organisé en novembre 2018 à l'Université de Bourgogne-Franche Comté.

9. On retrouve ici l'idée de la "segmentation précaire» de la bande dessinée, notamment dans le passage au roman graphique. Voir Baroni (2018).

10. On peut avoir un aperçu de la diversité des styles de dessin en parcourant le fil du compte Twitter "Random Sandman", qui publie quotidiennement des planches de l'œuvre. URL : https://twitter.com/randomsandman?lang=fr. En excluant les volumes dérivés de la série (The Dream Hunters et The Sandman: Overture), on arrive à un total de vingt-cinq dessinateurs ayant contribué à Sandman. 
11. L'arc Fables and Reflections est celui qui présente le moins d'unité de l'ensemble de Sandman; les sept chapitres y ont été réunis sous un même titre dans l'édition en recueil de DC Comics. L'épisode 50 a bien été écrit en même temps que les 29, 30 et 31 mais a été publié en périodique en retard, pour des raisons techniques. Par ailleurs, le degré d'indépendance des chapitres de la colonne de droite par rapport à l'intrigue principale varie fortement, ce que le schéma n'indique pas; certains n'ont aucune relation directe avec l'histoire, d'autres permettent d'expliquer certains éléments de l'intrigue.

12. On trouve une analyse détaillée de World's End dans Bernard (2016).

13. Selon la tripartition narrative proposée par Groensteen $(2001: 104,106)$, dans laquelle le récitant est l'instance qui parle dans les récitatifs.

\section{RÉSUMÉS}

L'objectif de cet article est d'étudier le chapitrage du comic book de Neil Gaiman Sandman (1989-1996) à partir de la dialectique entre les contraintes éditoriales de DC Comics et la flexibilité esthétique du roman graphique. On cherchera à montrer que ces deux tendances opposées aboutissent à la création d'une architecture complexe, dont l'objectif premier est de créer un espace d'archive de chaque genre littéraire, à mi-chemin entre production populaire et commentaire métatextuel.

This paper focuses on the chaptering of Neil Gaiman's comic book Sandman (1989-1996) through the dialectic between DC Comics' editorial constraints and the aesthetic flexibility of the graphic novel. We highlight that these two opposite trends lead to a complex architecture whose primary objective is to incorporate every literary genre in a new space, midway between popular production and metaxtual commentary.

\section{INDEX}

Mots-clés : chapitre, roman graphique, comic book, Sandman, Gaiman (Neil), bande dessinée Keywords : chapter, graphic novel, comic book, Sandman, Gaiman (Neil)

\section{AUTEUR}

RAPHAËL LUIS

ENS de Lyon, CERCC 